\title{
Improved Physics in SIMNRA 7
}

\author{
M. Mayer \\ Max-Planck-Institut für Plasmaphysik, EURATOM Association, Boltzmannstr. 2, \\ D-85748 Garching, Germany
}

\begin{abstract}
SIMNRA is an analytical code for the simulation of ion beam analysis energy spectra obtained by Rutherford backscattering, non-Rutherford scattering, elastic recoil detection analysis, and nuclear reaction analysis. Improvements of the simulation physics in SIMNRA version 7 include among others the skewness of all energy spread distributions, improved handling of scattering or reaction cross-sections with structure, generalized layer roughness, and sample porosity.
\end{abstract}

\section{Introduction}

In most cases the quantitative evaluation of ion beam analysis spectra obtained by Rutherford backscattering spectrometry (RBS), elastic (back-)scattering spectrometry (EBS), elastic recoil detection analysis (ERDA) and nuclear reaction analysis (NRA) requires computer simulation of the spectra in order to obtain the elemental composition and depth profiles of individual elements in the near-surface layer. Exceptions may be very thin layers, trace elements with non-overlapping signals [1], or spectra obtained by heavy-ion ERDA which can be converted directly into depth profiles without simulation [2].

A number of different codes has been developed during the last decades for the computer simulation of ion-beam analysis spectra $[3,4,5]$. These can be roughly divided into Monte-Carlo codes and analytical codes.

Monte-Carlo codes, such as TRIM.SP [6], SDTrimSP [7], SRIM [8,9,10,11,12], MCERD [13,14] or CORTEO [15], follow trajectories of individual particles in binary collision approximation. These codes are principally usable at energies from a few $100 \mathrm{eV}$ until many $\mathrm{GeV}$ (although the actual usable energy range of a specific code is often limited by computing time constraints, approximations for improving computing speed, or limited implementations of interaction potentials or stopping powers) and are very versatile. Multiple small- and large angle scattering effects are always included as well as complicated detection 
geometries or special (for example large or curved) detectors. Main drawback is the slow computing speed, which makes the use of these codes for routine analysis work of $\mathrm{MeV}$ ion beam analysis spectra problematic.

Analytical codes, such as SIMNRA [16,17], RUMP [18], or NDF [19], use a different approach: They calculate the evolution of energy distributions of ensembles of particles. Particle trajectories are approximated as straight lines, where multiple small-angle scattering effects or geometrical spread due to finite detector sizes are added as (typically small) additional corrections to the energy distributions. Those approximations make these codes only applicable if multiple scattering effects are sufficiently small, i.e. if particle energies are sufficiently high. The dual-scattering approximation extends the applicability of these codes towards lower energies [20] at the cost of computing time. Main advantage of these types of codes is a very fast computation and the maturity of the codes [4].

SIMNRA version 6 is a popular code for the simulation of ion beam analysis spectra. The initial release of SIMNRA 6.0 was in the year 2006. Since its initial release a number of bug fixes and minor improvements were released in versions 6.01 to 6.06 . The physics basis, however, remained unchanged. New physics was implemented in version 6.10 and higher and was partly described in a number of publications [21,22,23,24], but these versions never became publicly available. SIMNRA 7 summarises all improvements of the simulation physics accumulated during the last years. This improved physics is shortly summarised in this paper, details can be found in the given references.

\section{Skewness of energy distributions}

The slowing down of swift ions in matter is always associated with energy spread due to energy-loss straggling [25] and multiple small-angle scattering $[26,27,28]$. An accurate calculation of the shape of electronic energy-loss straggling distributions is possible within the framework of a general stopping and straggling theory, such as the Bohr, Bethe, or Bloch theory [29,30,25]. This requires an additional numerical convolution, which increases the computing time by about one order of magnitude: This is impractical due to computing time restrictions in simulation codes. Moreover, the shape of the straggling distribution cannot be calculated if empirical or semi-empirical stopping or straggling models (such as SRIM stopping powers [10] or Yang straggling [31]) are used, because these models provide only information about one specific moment of the energy distribution: A stopping power model gives the mean energy loss and mean energy, a straggling model the variance of the straggling. But it is usually impossible to get information about higher moments (such as the skewness and kurtosis) from such semi-empirical models. 
Because these models often provide better accuracy than a general stopping and straggling theory, such semi-empirical models are often used in practice.

The different energy spread distributions relevant for IBA (electronic energyloss straggling, geometrical straggling, multiple scattering, energy spread due to porosity) can be approximated with sufficient accuracy by two-piece normal distributions (TPNDs) [21]. This slows down calculations only marginally and provides energy distributions with correct mean value, variance and third moment [21], thus resulting in higher accuracy in spectrum simulation. From the different classes of skewed Gaussian-type functions the TPND has been selected due to its good compatibility with the calculational schemes for IBA.

As was already shown in [29], the first three moments are sufficient for an accurate approximation of the energy-loss distributions if the energy-loss is not too small. We obtain the mean value from the energy loss due to the stopping power $[32,33,34,35,10]$ and the variance from a straggling theory, such as [31]. As third moment we use nonrelativistic free Coulomb scattering [25, chapter 8.11]. Due to the very limited availability of experimental data for third moments the accuracy of the free scattering theory is hard to judge. At sufficiently large ion velocities it should describe the third moment comparably accurate as Bohr theory describes straggling, while at lower ion velocities free scattering will overestimate the third moment.

Measured energy distributions of $19.68 \mathrm{MeV}$ protons after traversing $0.2675 \mathrm{~g} / \mathrm{cm}^{2}$ and $0.497 \mathrm{~g} / \mathrm{cm}^{2}$ aluminum are shown in Fig. 1. The energy distributions are better approximated by the SIMNRA 7 model. The approximation by Gaussian energy distributions in SIMNRA 6 gives the correct widths (FWHMs) of the distributions, but the shape is not as well reproduced.

Skewed energy distributions have been also implemented for multiple-scattering energy distributions [21], geometrical spread, and energy spread by porosity [24]. Comparisons to existing experimental data or Monte-Carlo simulations using the MCERD code can be found in [21].

\section{Cross-sections with structure}

SIMNRA uses the classical brick concept for calculating the number of scattered $^{1}$ particles [17, Fig. 1]. This requires the calculation of the (mean) cross-sections at the beginning and end of each brick. The energy distribution of an ensemble of straggled particles can be described by a distribution

\footnotetext{
1 For simplicity the term 'scattered' will be used throughout this section. However, a 'scattered' particle may be also a recoil or a nuclear reaction product.
} 


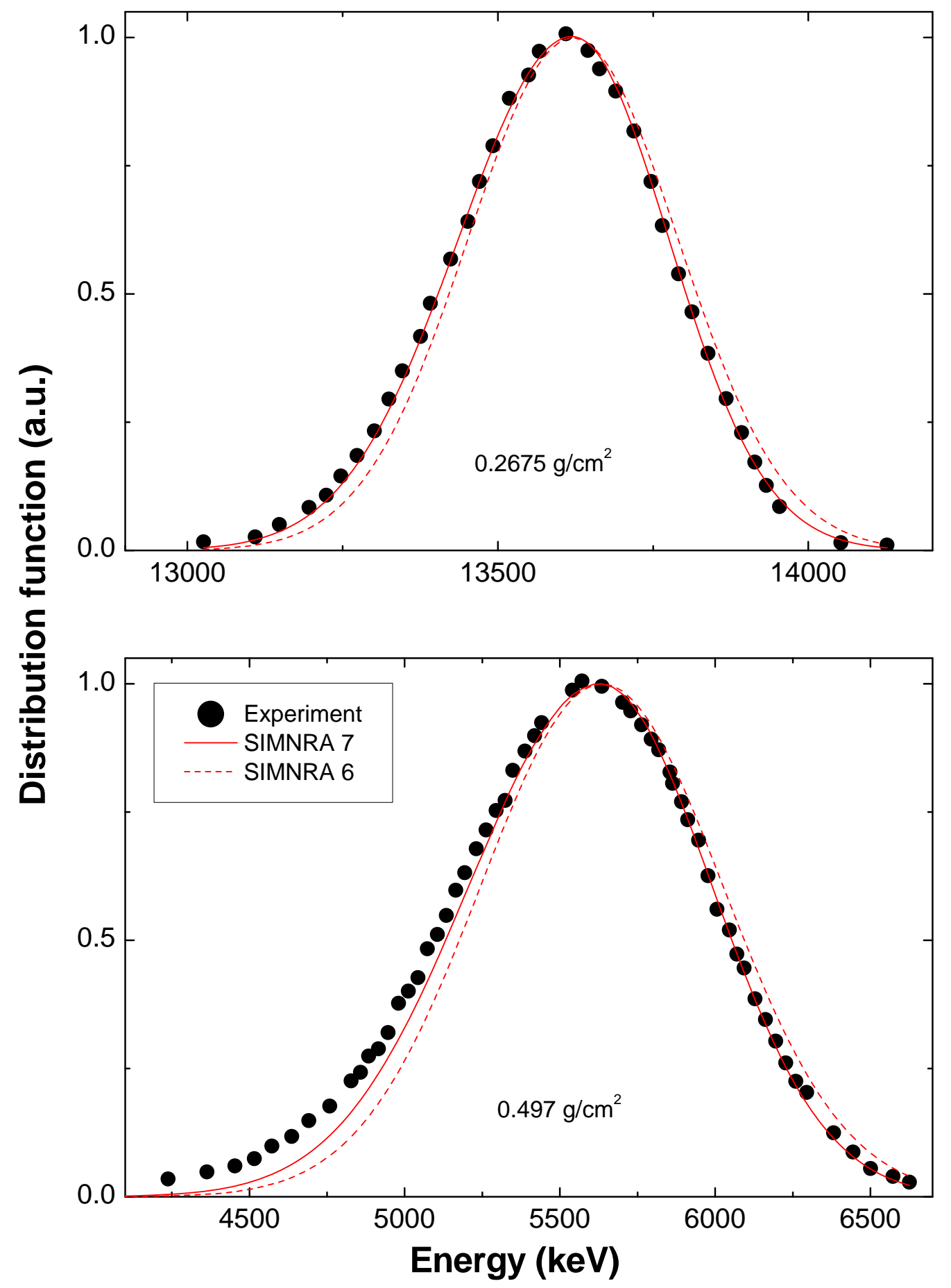

Fig. 1. Energy distributions of $19.68 \mathrm{MeV}$ protons after traversing $0.2675 \mathrm{~g} / \mathrm{cm}^{2}$ and $0.497 \mathrm{~g} / \mathrm{cm}^{2}$ aluminum. Dots: Experimental data from [47]; Solid line: SIMNRA 7 calculation using a two-piece normal distribution for electronic energy-loss straggling; Dashed line: SIMNRA 6 calculation using a Gaussian energy distribution. 
function $f(E)$, where $f(E)$ is typically close to Gaussian and normalized to $\int f(E) d E=1$. A mean cross-section $\bar{\sigma}$ for this energy distribution then can be defined by

$$
\bar{\sigma}=\int f(E) \sigma(E) d E
$$

with $\sigma(E)$ the energy-dependent scattering cross-section.

SIMNRA 6 uses the simple approximation $\bar{\sigma}=\sigma(\bar{E})$, with $\bar{E}$ the mean energy of the energy distribution: $\bar{E}=\int E f(E) d E$. This allows a very fast computation and is sufficiently accurate for slowly varying cross-sections such as Rutherford cross-section. As has been shown in [36], however, this approximation gets inaccuate for cross-sections with structure (such as resonant cross-sections for scattering of nuclear reactions), if the energy spread of the straggling distribution gets comparable or larger than the width of structures in the cross-section. SIMNRA 7 evaluates eq. 1 by numerical integration using the energy distribution $f(E)$ as described in section 2 .

Moreover, the shape of the cross-section influences not only the number of scattered particles via the mean cross-section, but influences also the shape of the energy distribution of the ensemble of particles after scattering. If a particle had the energy $E$ before scattering, then the energy $E^{\prime}$ after scattering is given by $E^{\prime}=K E$, with $K$ the kinematic factor. For a constant (or slowly varying) cross-section the energy distribution after scattering $f^{\prime}\left(E^{\prime}\right)$ is then simply given by $f^{\prime}\left(E^{\prime}\right)=(1 / K) f(E)$, which has mean value $\bar{E}^{\prime}=K \bar{E}$, variance $V^{\prime}=K^{2} V$ and third central moment $M^{\prime}=K^{3} M$, with $V$ the variance and $M$ the third central moment of the energy distribution before scattering. For a cross-section with structure this has to be modified to $f^{\prime}\left(E^{\prime}\right) d E^{\prime}=(1 / \bar{\sigma}) f(E) \sigma(E) d E$, i.e. the shape of the energy distribution after scattering is influenced by the energy dependence of the cross-section: A Gaussian energy distribution before scattering will usually become nonGaussian after scattering, if the cross-section has narrow structures. The first three moments of the energy distribution after scattering are then given by

$$
\begin{aligned}
\bar{E}^{\prime} & =K / \bar{\sigma} \int E f(E) \sigma(E) d E \\
V^{\prime} & =K^{2} / \bar{\sigma} \int(E-\bar{E})^{2} f(E) \sigma(E) d E \\
M^{\prime} & =K^{3} / \bar{\sigma} \int(E-\bar{E})^{3} f(E) \sigma(E) d E .
\end{aligned}
$$

SIMNRA 7 uses numerical integration in order to calculate the first three moments of the energy distribution after scattering using eqs. 2 to 4 . This increases the computing time by about $30 \%$. The energy distribution is then 


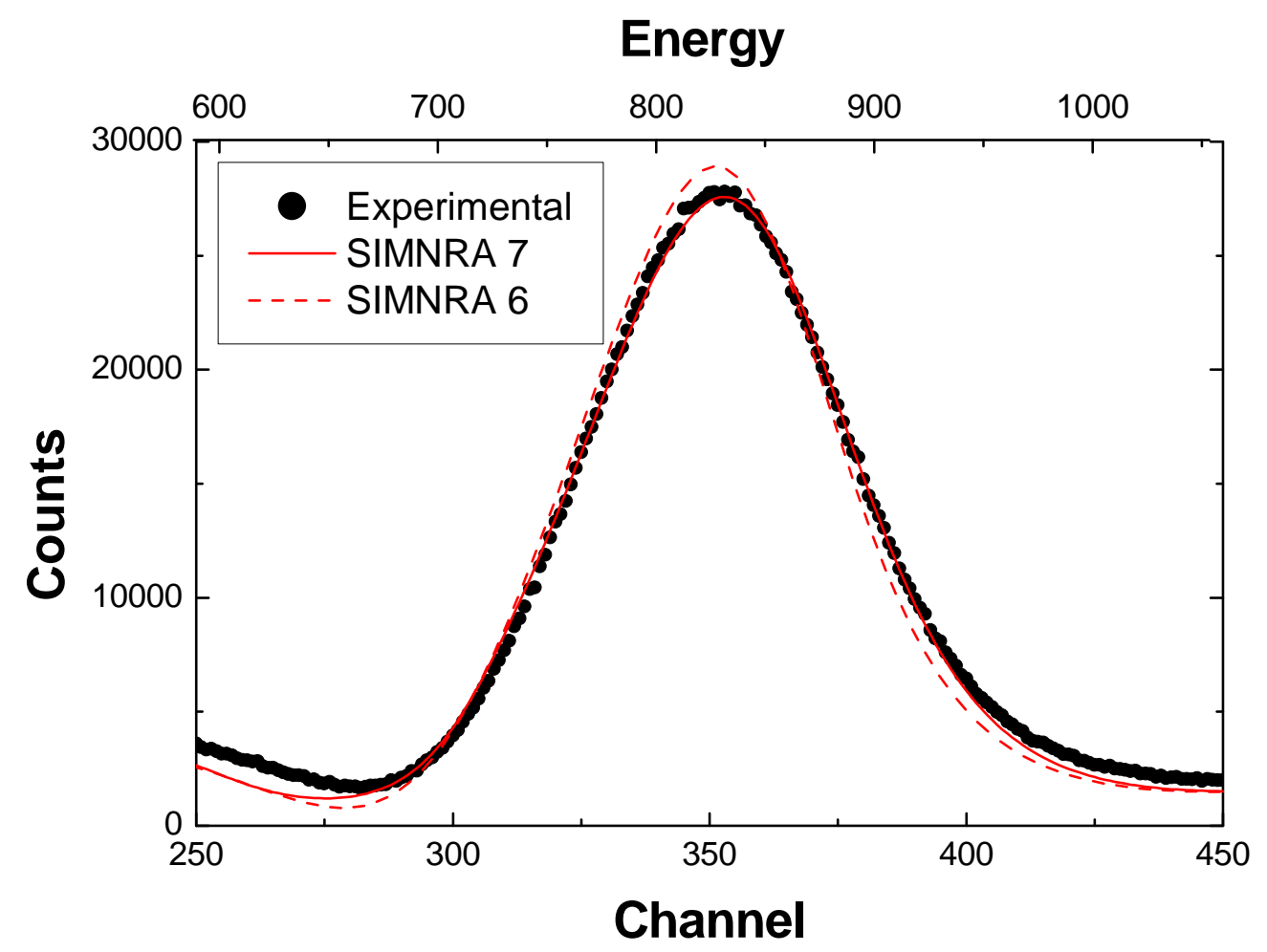

Fig. 2. Backscattering of $2000 \mathrm{keV}$ protons from pyrolytic graphite at a scattering angle of $165^{\circ}$. Dots: Experimental data; Solid line: Simulation with SIMNRA 7 using the improved treatment of resonant cross-sections described in section 3; Dashed line: Simulation using SIMNRA 6. The SigmaCalc cross-section from [37] was used for both simulations.

propagated to the surface using the methods described in section 2 . This procedure takes the first three moments of the energy distributions into account.

This change of the shape of the energy distribution by the scattering crosssection has been already pointed out in [36]. However, the procedure described in [36] is somewhat inconsequent, because the shape of the energy distribution is assumed to be always Gaussian, and only the mean cross-section according to eq. 1 and the change of the mean energy according to eq. 2 is taken into account.

The energy spectrum of $2000 \mathrm{keV}$ protons backscattered from pyrolytic graphite is shown in Fig. 2. The spectrum is dominated by the well-known resonance in the cross-section at about $1740 \mathrm{keV}$. The simulation uses the SigmaCalc cross-section from [37]. The simulation with SIMNRA 6 gives a a resonance peak, which is too narrow and too high, while SIMNRA 7 perfectly reproduces the shape of the peak. It should be noted, though, that for bulk samples SIMNRA 6 still yields the correct total number of counts (i.e. the integrated number of counts for the peak is almost identical for SIMNRA 6 and SIMNRA 7), but the width of the simulated peak is usually too narrow. 


\section{Generalized layer roughness}

Rough layers are characterised by a distribution of layer thicknesses. The SIMNRA 6 model of layer roughness assumes a $\Gamma$-distribution of layer thicknesses [38]. Initially the $\Gamma$-distribution was an ad-hoc proposal, because it converges towards a Gaussian distribution if the width of a distribution is smaller than its mean value, but, in contrast to a Gaussian distribution, it is positive definite. Although initially ad-hoc, this model turned out to give a very good description for a large number of different rough layered systems with 'natural' roughness.

However, in a number of cases this roughness model is insufficient. It does not describe partial coverage of a surface, for example of plasma-eroded surfaces [39] or meshes, and it does not describe artificial surfaces where defined variations of layer thicknesses are used, for example gratings [40,23,22] or nano-dots. SIMNRA 7 allows to use arbitrary layer thickness distributions: These can be supplied through an input file which contains the frequency distribution of the layer thicknesses. Correlations between incident and exit beam (for example incidence through a valley and exit through a hill) are neglected: This approximation is accurate in transmission geometry and at backward scattering angles close to $180^{\circ}$, but gets more and more approximate if other angles are used. This model is therefore well suited for backscattering analysis at scattering angles larger than about $160^{\circ}$ for layers, where the layer thickness is smaller than the lateral scale of thickness variations. For 2-dimensional samples usually a sample orientation, where these approximations are precisely fulfilled, can be found [23].

The frequency distribution of layer thicknesses measured by ion beam analysis generally does not allow unambiguously to reconstruct the lateral distribution of thicknesses, i.e. the surface profile. However, with the help of additional knowledge, for example about the periodicity of periodic structures and knowledge about symmetries, even the lateral distribution can be reconstructed in some cases. The evolution of the shape of a sputter-eroded silicon grating during the sputtering process can be found in [23].

\section{Porous layers}

Porous materials are characterised by a mixture of material and voids. Incident ions traverse a random number of voids: If an ion crosses a larger number of voids, then its energy loss will be smaller than that of an ion which traverses a larger path length in the material. A random distribution of pores therefore results in a distribution of path lengths in the material and consequently in 
a distribution of energy losses. The resulting energy spread can be measured and can be used to derive information about the porosity of a layer [24].

Because ion trajectories at sufficiently high energies are close to straight lines, ion beam analysis provides information about the path length distribution function in the material forming a porous layer. The path length distribution function approaches rapidly a Gaussian, if more than very few pores are traversed. Consequently, ion beam analysis can provide the mean path length in the material and the variance of the path length distribution function for a porous layer. Both numbers increase linearly with layer thickness. The variance per unit length is therefore a fundamental number characteristic for the geometrical arrangement of pores in a porous layer.

The variance of the path length distribution per unit lenth can be derived directly from a measurement without a model. However, usually a different information, for example about the pore volume fraction or about the diameter of pores, is seeked. This requires a model which connects the measured variance per unit lenth with the geometrical arrangement of pores. SIMNRA 7 implements the simplest possible model of a porous layer: It assumes a random arrangement of overlapping equally-sized spherical pores in tree dimensions [24]. The statistical properties (variances and third central moments) of the path length distribution functions of these porous structures have been computed by Monte-Carlo simulations and are tabulated in the program. The variance per unit length depends on pore volume fraction and on pore diameter. If the variance per unit length has been measured by ion beam analysis and if the pore volume fraction is known (it can be determined for example by combining Rutherford backscattering and focused ion beam cross-sectioning), then the pore diameter can be determined.

The surface of a porous $\mathrm{V}_{2} \mathrm{O}_{5}$ layer with $40 \%$ pore volume fraction is compared to the SIMNRA 7 computational model in Fig. 3. The computational model is a three-dimensional cube, the figure shows the section of this cube with the central plane. The diameter of the spherical pores is $31 \mathrm{~nm}$. These form larger, irregularly shaped elongated pores by random overlap. The computational model assumes a fully random distribution of the pores. In reality, porous materials have a large degree of randomness, but they are not fully random.

An RBS spectrum of the porous $\mathrm{V}_{2} \mathrm{O}_{5}$ layer from Fig. 3 is shown in Fig. 4 . The low-energy edges of the $\mathrm{V}$ and $\mathrm{O}$ signals are clearly broadened by the porosity. The computer simulation assuming a porous layer is in very good agreement with the experimental data and yields a variance of the path length distribution per unit length of $4.6 \mathrm{~nm}^{2} / \mathrm{nm}$. For a porosity volume fraction of $40 \%$ this results in a pore diameter of $31 \mathrm{~nm}$. This number is in good agreement with the SEM image in Fig. 3. 

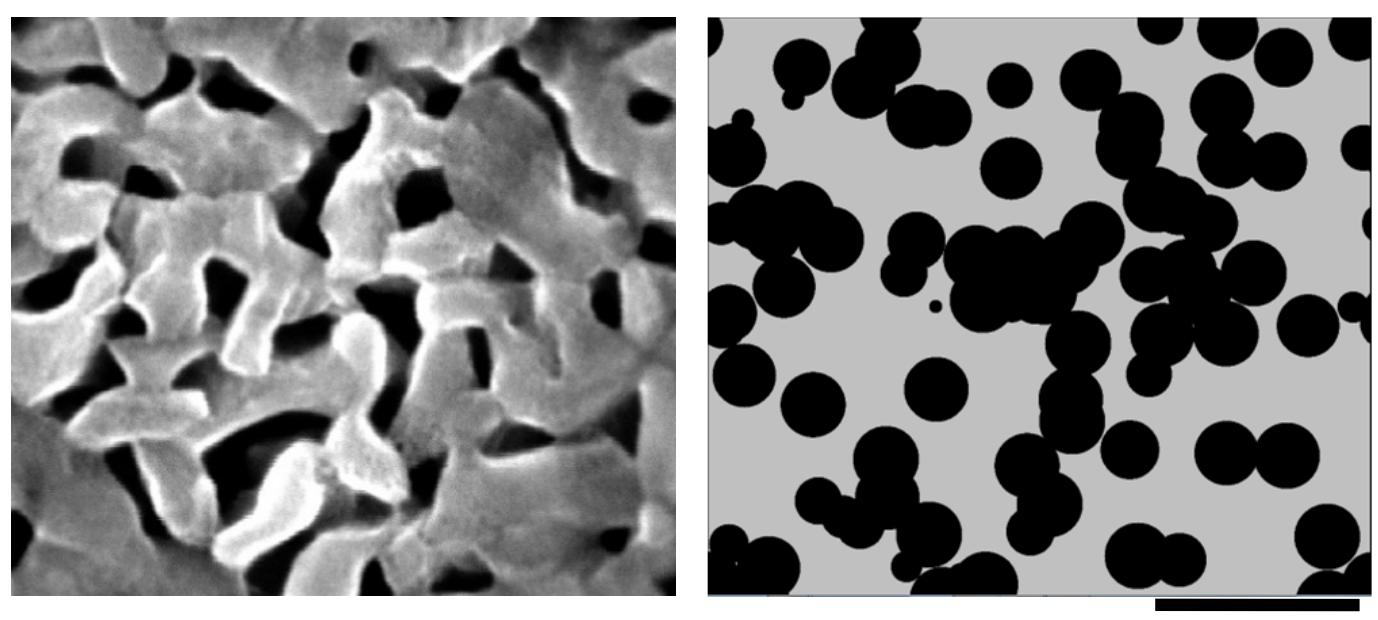

$100 \mathrm{~nm}$

Fig. 3. Left: Scanning electron micrograph of the surface of a porous $\mathrm{V}_{2} \mathrm{O}_{5}$ layer. Right: Cross-section through the central plane of the computational model of the porous layer. $\mathrm{V}_{2} \mathrm{O}_{5}$ material is grey, pores in the material are black. The pore diameter is $31 \mathrm{~nm}$.

It should be kept in mind, that porosity and layer roughness may have identical effects: Both result in a broadening of the low-energy edge of a porous or rough layer. This ambiguity is difficult to resolve with ion beam analysis methods alone and may require additional knowledge about the samples, for example from scanning electron microscopy or focused ion beam cross-sectioning.

\section{Other physics improvements}

- Window for external beam: A window for an external beam has been included in SIMNRA 7. The window is placed between the incident beam and the target and acts through its stopping and straggling. It can consist of an arbitrary number of layers, which may contain roughness or porosity.

- Energy-dependent detector sensitivity: An energy-dependent detector sensitivity can be supplied.

- Universal scattering cross-section: The scattering cross-section based on the universal screened potential can be selected instead of screened Rutherford cross-sections. The pre-calculated high-precision cross-section data from [41] are used. The universal scattering cross-section is mainly useful at very low energies, as are typical for medium energy ion scattering (MEIS). 


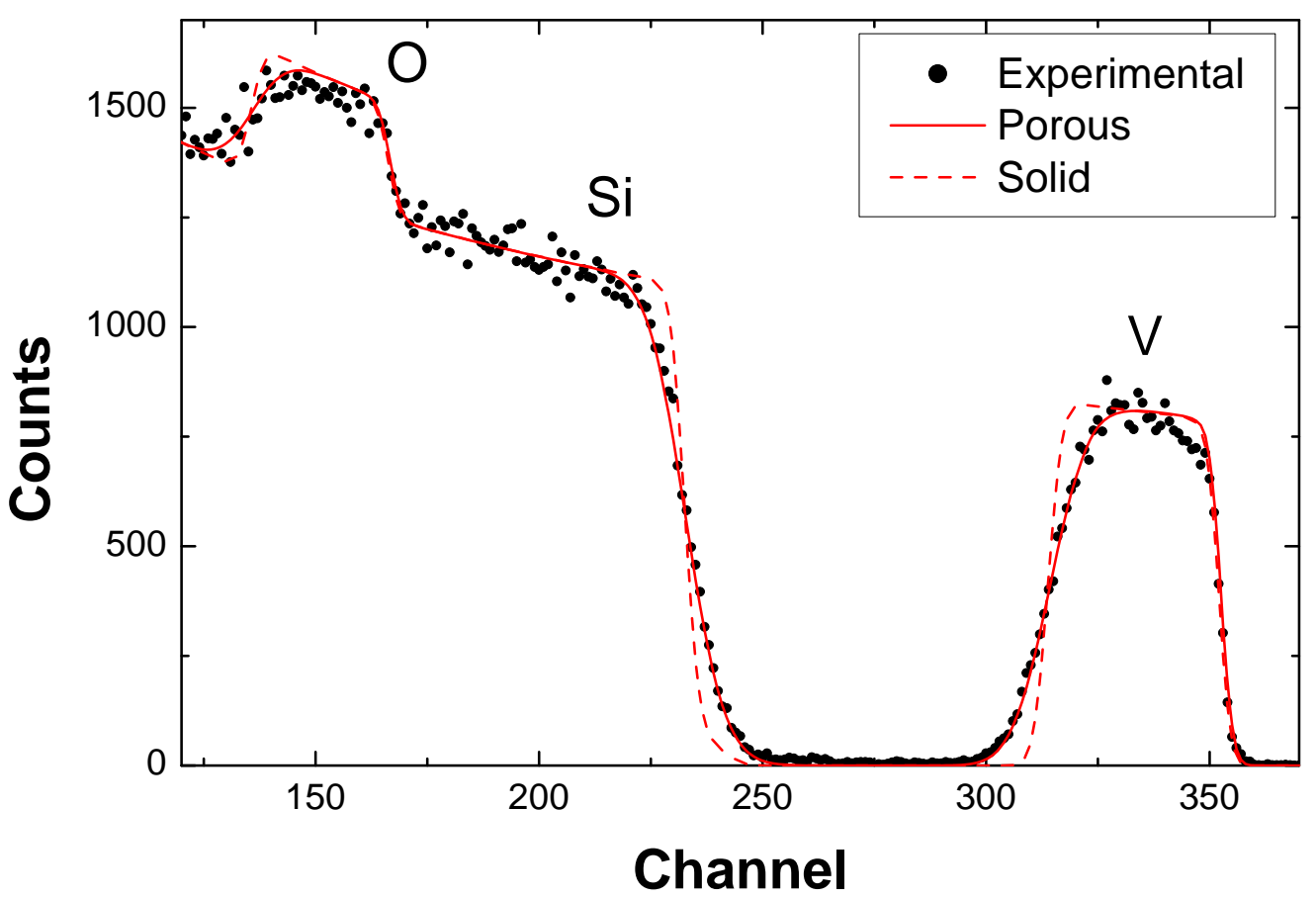

Fig. 4. RBS spectrum of a porous $\mathrm{V}_{2} \mathrm{O}_{5}$ layer on silicon substrate using $1500 \mathrm{keV}{ }^{4} \mathrm{He}$ ions at a scattering angle of $165^{\circ}$. Dots: Experimental data; Solid line: Computer simulation assuming a porous layer with $40 \%$ pore fraction and a pore diameter of $31 \mathrm{~nm}$; Dashed line: Computer simulation assuming a dense layer.

\section{Programming support}

SIMNRA 7 uses the IBA data format (IDF) [42] as its native file format for storing spectra and all input data. The file format is called xnra and is somewhat more restrictive than IDF. Nevertheless, xnra files are always valid IDF files. IDF is a well-defined and documented XML-based file format for storing ion beam analysis data and allows easy exchange of data between different ion beam analysis programs.

All SIMNRA objects can be accessed through OLE automation. This allows the development of new codes, which make use of SIMNRA as simulating kernel. This possibility has been already used for the development of extended target editors [43,44], depth resolution calculations [45], or evaluation of depth profiles using Bayesian analysis [46]. In SIMNRA 6 most (but not all) objects could be accessed by OLE automation. In SIMNRA 7 all properties of all objects can be accessed by external programs. All OLE automation methods are backward compatible, i.e. programs written for SIMNRA 6 should work also for SIMNRA 7. 


\section{Additional programs in the SIMNRA package}

All details of SIMNRA calculations can be inspected using the program VIEWNRA, allowing a detailed inspection of energy loss calculations, straggling evaluations, evolution of angular spread due to multiple scattering etc. This program was initially developed as a debugging tool, but was made publicly available in order to provide full transparency about all details of SIMNRA simulations. This can be used for inspecting details of calculations, or for teaching purposes.

RESOLNRA [45] calculates the achievable depth resolution for a given experiment, and allows to optimize the experimental conditions (incident energy and angle) in order to achieve an optimal depth resolution. RESOLNRA uses SIMNRA for all calculations and treats RBS, ERDA and NRA. It includes the energy broadening contributions due to electronic energy-loss straggling, geometrical straggling, multiple scattering, absorber foils, detector resolution and sample porosity. Additional constraints, such as minimum or maximum possible beam energies, limitations of incident or exit angles, or requirements for a specific projectile energy in a specific depth, can be taken into account, thus allowing to find the optimum experimental conditions under these additional experimental constraints. The user interface was developed in order to allow a quick optimization of the experimental conditions. The use of identical input files by RESOLNRA and SIMNRA offers the possibility of a rapid experimental design/experimental data evaluation cycle. RESOLNRA 1.5 allows to access all depth resolution calculations through OLE automation.

SIMNRA 7 works together with the cross-section calculator SigmaCalc. For many ion-target combinations theoretical SigmaCalc cross-sections are available by default.

\section{Conclusions}

The program package SIMNRA 7 improves the simulation physics model of the current version SIMNRA 6 by taking the skewness of all energy spread distributions into account, and by an improved handling of cross-sections with structure. The applicability of the code towards lower energies has been extended by adding the Universal scattering cross-section. More complex experimental set-ups can be directly simulated by the addition of an external window and energy-dependent detector sensitivity, and more complex samples can be simulated by introducing generalized layer roughness and sample porosity. Programming support and interoperability with other programs has been improved by providing full access to all objects and properties by OLE 
automation and the use of the IBA data format as native file format for storing all data. The additional programs VIEWNRA, RESOLNRA and SigmaCalc allow to inspect all details of SIMNRA calculations, allow to perform depth resolution calculations, and allow an easy use of non-Rutherford scattering cross-sections.

\section{Acknowledgements}

The technical support of J. Dorner and M. Fusseder with all ion beam measurements and electron microscopy by G. Matern is gratefully acknowledged. The porous $\mathrm{V}_{2} \mathrm{O}_{5}$ sample was provided by O. Dubreuil and F. Mathis, University of Liege, Belgium.

\section{References}

[1] P. Børgesen, R. Behrisch, and B.M.U. Scherzer. Appl. Phys. A 111 (1982) 717.

[2] A. Bergmaier, G. Dollinger, and C.M. Frey. Nucl. Instr. Meth. B 99 (1995) 488.

[3] E. Rauhala, N.P. Barradas, S. Fazinic, M. Mayer, E. Szilágyi, and M. Thompson. Nucl. Instr. Meth. B 244 (2006) 436. doi: 10.1016/j.nimb.2005.10.024.

[4] N.P. Barradas, K. Arstila, G. Battistig, M. Bianconi, N. Dytlewski, C. Jeynes, E. Kótai, G. Lulli, M. Mayer, E. Rauhala, E. Szilágyi, and M. Thompson. Nucl. Instr. Meth. B 262 (2007) 281. doi: 10.1016/j.nimb.2007.05.018.

[5] N.P. Barradas, K. Arstila, G. Battistig, M. Bianconi, N. Dytlewski, C. Jeynes, E. Kótai, G. Lulli, M. Mayer, E. Rauhala, E. Szilágyi, and M. Thompson. Nucl. Instr. Meth. B 266 (2008) 1338. doi: 10.1016/j.nimb.2007.10.043.

[6] W. Eckstein. Computer Simulation of Ion-Solid Interactions, vol. 10 of Materials Science. Springer, Berlin, Heidelberg, New York, 1991.

[7] W. Eckstein, R. Dohmen, A. Mutzke, and R. Schneider. SDTrimSP: A MonteCarlo code for calculating collision phenomena in randomized targets. Tech. Rep. IPP 12/3, Max-Planck-Institut für Plasmaphysik, Garching, 2007.

[8] J.P. Biersack and L.G. Haggmark. Nucl. Instr. Meth. 174 (1980) 257.

[9] J.P. Biersack and W. Eckstein. Appl. Phys. A34 (1984) 73.

[10] J.F. Ziegler. Nucl. Instr. Meth. B 219-220 (2004) 1027.

[11] J.F. Ziegler, M.D. Ziegler, and J.P. Biersack. Nucl. Instr. Meth. B 268 (2010) 1818. 
[12] J.F. Ziegler. SRIM: The stopping and range of ions in matter. http://www . srim.org/.

[13] K. Arstila, T. Sajavaara, and J. Keinonen. Nucl. Instr. Meth. B 174 (2001) 163.

[14] K. Arstila, J.A. Knapp, K. Nordlund, and B.L. Doyle. Nucl. Instr. Meth. B 219-220 (2004) 1058.

[15] F. Schiettekatte. Nucl. Instr. Meth. B 266 (2008) 1880.

[16] M. Mayer. SIMNRA user's guide. Tech. Rep. IPP 9/113, Max-Planck-Institut für Plasmaphysik, Garching, 1997.

[17] M. Mayer. SIMNRA, a simulation program for the analysis of NRA, RBS and ERDA. In Proceedings of the 15th International Conference on the Application of Accelerators in Research and Industry (Woodbury, New York, 1999), J. L. Duggan and I. Morgan, Eds., vol. 475 of AIP Conference Proceedings, American Institute of Physics, p. 541. doi: 10.1063/1.59188.

[18] R. Doolittle. Nucl. Instr. Meth. B 9 (1985) 344.

[19] N.P. Barradas. J. Phys. D: Appl. Phys. 34 (2001) 2109.

[20] W. Eckstein and M. Mayer. Nucl. Instr. Meth. B 153 (1999) 337. doi: 10.1016/S0168-583X(98)01011-8.

[21] M. Mayer, K. Arstila, and U. von Toussaint. Nucl. Instr. Meth. B 268 (2010) 1744. doi: $10.1016 /$ j.nimb.2010.02.057.

[22] M. Mayer, W. Eckstein, H. Langhuth, F. Schiettekatte, and U. von Toussaint. Nucl. Instr. Meth. B 269 (2011) 3006. doi: 10.1016/j.nimb.2011.04.066.

[23] H. Langhuth, M. Mayer, and S. Lindig. Nucl. Instr. Meth. B 269 (2011) 1811. doi: 10.1016/j.nimb.2011.05.002.

[24] M. Mayer, U. von Toussaint, J. Dewalque, O. Dubreuil, C. Henrist, R. Cloots, and F. Mathis. Nucl. Instr. Meth. B 273 (2012) 83. doi: 10.1016/j.nimb.2011.07.045.

[25] P. Sigmund. Particle Penetration and Radiation Effects, vol. 151 of Springer Series in Solid State Sciences. Springer, Berlin, Heidelberg, 2006.

[26] E. Szilágyi, F. Pászti, and G. Amsel. Nucl. Instr. Meth. B 100 (1995) 103.

[27] E. Szilágyi. Nucl. Instr. Meth. B 161-163 (2000) 37.

[28] G. Amsel, G. Battistig, and A. L'Hoir. Nucl. Instr. Meth. B 201 (2003) 325.

[29] C. Tschalär. Nucl. Instr. Meth. 61 (1968) 141.

[30] C. Tschalär. Nucl. Instr. Meth. 64 (1968) 237.

[31] Q. Yang, D.J. O'Connor, and Z. Wang. Nucl. Instr. Meth. B 61 (1991) 149. 
[32] H.H. Andersen and J.F. Ziegler. Hydrogen - Stopping Powers and Ranges in All Elements, vol. 3 of The Stopping and Ranges of Ions in Matter. Pergamon Press, New York, 1977.

[33] J.F. Ziegler. Stopping Cross-Sections for Energetic Ions in all Elements, vol. 5 of The Stopping and Ranges of Ions in Matter. Pergamon Press, New York, 1980 .

[34] J.F. Ziegler, J.P. Biersack, and U. Littmark. The Stopping and Range of Ions in Solids, vol. 1 of The Stopping and Ranges of Ions in Matter. Pergamon Press, New York, 1985.

[35] G. Konac, S. Kalbitzer, Ch. Klatt, D. Niemann, and R. Stoll. Nucl. Instr. Meth. B 136-138 (1998) 159.

[36] N.P. Barradas, E. Alves, C. Jeynes, and M. Tosaki. Nucl. Instr. Meth. B 247 (2006) 381.

[37] A.F. Gurbich. Nucl. Instr. Meth. B 136-138 (1998) 60.

[38] M. Mayer. Nucl. Instr. Meth. B 194 (2002) 177. doi: 10.1016/S0168$583 \mathrm{X}(02) 00689-4$.

[39] M. Mayer, V. Rohde, G. Ramos, E. Vainonen-Ahlgren, J. Likonen, J.L. Chen, and ASDEX Upgrade team. Physica Scripta T128 (2007) 106.

[40] I. Bizyukov, A. Mutzke, R. Schneider, and J. Davis. Nucl. Instr. Meth. B 268 (2010) 2631.

[41] H.G. Schlager and W. Eckstein. The scattering integrals - integration and accuracy. Tech. Rep. IPP 9/69, Max-Planck-Institut für Plasmaphysik, Garching, 1991.

[42] N.P. Barradas, M. Mayer, and M. Thompson. Nucl. Instr. Meth. B 268 (2010) 1824. doi: 10.1016/j.nimb.2010.02.093.

[43] M. J. F. Healy, M. Torres, and J. D. Painter. Nucl. Instr. Meth. B 249 (2006) 789 .

[44] J.L. Colaux. Simtarget. http://webapps .fundp.ac . be/physique/simtarget/.

[45] M. Mayer. Nucl. Instr. Meth. B 266 (2008) $1852 . \quad$ doi: 10.1016/j.nimb.2007.11.071.

[46] K. Schmid and U. von Toussaint. Nucl. Instr. Meth. B 281 (2012) 64.

[47] C. Tschalär and H.D. Maccabee. Phys. Rev. B1 (1970) 2863. 